\title{
Six-Week Online Multi-component Positive Psychology Intervention Improves Subjective Wellbeing in Young Adults
}

\author{
Miranda R. Chilver ${ }^{1,2}$. Justine M. Gatt ${ }^{1,2}$ (D)
}

Accepted: 26 August 2021 / Published online: 5 September 2021

(c) The Author(s), under exclusive licence to Springer Nature B.V. 2021

\begin{abstract}
Improving mental wellbeing has a range of benefits for society, including increased productivity, longevity, and resiliency. However, interventions designed to improve mental wellbeing are often only compared to waitlist controls, leaving uncertainty regarding the mechanisms of their effectiveness. The current study in 326 participants assessed a sixweek positive psychology intervention against an active control $(n=163)$ in an online randomized control trial. Outcome measures included life satisfaction, wellbeing (subjective and psychological wellbeing), stress, depression and anxiety symptoms, and selfcompassion. The potential moderating effect of participating during the ongoing COVID19 pandemic was also explored. The intervention group showed greater improvements in life satisfaction by week six $(\beta=0.18, p=.014)$ and were maintained through to 7 weeks post-baseline $(\beta=0.23, t=3.07, p=.002)$ and remained significant when accounting for COVID-19 restrictions. An improvement in composite wellbeing from baseline to 7 weeks post-baseline was detected when accounting for COVID-19 restrictions. Composite wellbeing and total depression and anxiety symptoms improved significantly more in the intervention group for participants with low baseline resiliency resources. These findings support the efficacy of using online multi-component positive psychology interventions in boosting wellbeing and reducing distress symptoms particularly in individuals with fewer resiliency resources who may need added support.
\end{abstract}

Keywords COVID-19 $\cdot$ Randomized control trial $\cdot$ Resilience $\cdot$ COMPAS-W $\cdot$ Stress

\section{Introduction}

Mental wellbeing can be defined as a state of optimal positive functioning that is related but independent from mental illness (World Health Organization, 2004). States of mental wellbeing are not only desirable in the general population (Parks et al., 2012; Sanders et al., 2019), but also associated with increased longevity (Martín-María et al., 2017) and health (Kushlev et al., 2020), improved work productivity (Gandy et al., 2016; Hamar et al.,

Justine M. Gatt

j.gatt@neura.edu.au

1 Neuroscience Research Australia, Barker St, Randwick, Sydney, NSW 2031, Australia

2 School of Psychology, University of New South Wales, Sydney, NSW 2052, Australia 
2015), and resilience against mental illness (Grant et al., 2013; Iasiello et al., 2019; Lamers et al., 2015). Many people actively seek out methods to improve their mental wellbeing (Parks et al., 2012; Sanders et al., 2019). Developing evidence-based interventions that are accessible to the general population is important to help individuals promote their own wellbeing and improve life satisfaction, health, and productivity of the general population.

A range of interventions including acts of kindness, gratitude, positive reminiscence, and self-compassion have been proposed to improve mental wellbeing (for review see Sin \& Lyubomirsky, 2009). Collectively, these interventions have been referred to as positive psychology interventions (PPIs). Some activities have a stronger evidence base than others. Acts of kindness interventions, for instance, have been found to have a moderate effect size (Cohen's $d=0.28$ ) on subjective wellbeing in a meta-analysis of 27 studies with over 4000 participants (Curry et al., 2018). Another popular PPI, gratitude, has been found to have a similar effect size $(d=0.31)$ when compared against a measurement-only control condition, but a much smaller effect size $(d=0.14)$ when compared against a matched control activity (Davis et al., 2016). Some other interventions have a smaller research base but show promising outcomes. Positive reminiscence is a psychological intervention commonly used in elderly samples, often applied in nursing homes (Meléndez Moral et al., 2015; Pinquart \& Forstmeier, 2012). In these samples, the effect size has also been moderate (Hedge's $g=0.33$ ) for measures of positive wellbeing based on a meta-analysis of 128 studies (Pinquart \& Forstmeier, 2012). The benefits found in older adults appear to generalise to younger samples based on a study of young adults (Hallford \& Mellor, 2016). Relative to these other interventions, self-compassion interventions are reported to have a moderate effect size (Pearson's $r=0.47$ ) on overall wellbeing in a meta-analysis including 79 samples and over 16,000 participants (Zessin et al., 2015). It is noted that while the observed effect of self-compassion was strong across studies, studies included in the metaanalysis were not required to have a control condition. Nonetheless, each of these interventions show great promise in their effectiveness.

While the aforementioned interventions showed significant positive effects on mental wellbeing on their own, it has been suggested that providing a variety of activities, rather than just one, improves their overall effectiveness (Schueller \& Parks, 2012). Variety may make PPIs more appealing to users, resulting in higher engagement and efficacy (Schueller \& Parks, 2012). Furthermore, the application of multi-component PPIs (MPPIs) is theorised to reduce hedonic adaptation, improving their effectiveness over time (Bao \& Lyubomirsky, 2014; Lyubomirsky, 2012). A meta-analysis of 50 randomized control trials on multi-component PPIs found them to be effective in improving both subjective $(g=0.34)$ and psychological $(g=0.39)$ wellbeing (Hendriks et al., 2020), with a particular advantage in improving psychological wellbeing over single-component PPIs with effect sizes of $d=0.34$ and $d=0.20$ reported for subjective and psychological wellbeing, respectively (Bolier et al., 2013).

Although these meta-analyses all provide evidence for the efficacy of PPIs, it is unclear whether online interventions can achieve the same results as face-to-face interventions, and whether PPIs are more effective than other non-evidence-based activities due to a placebo effect. For instance, one of the largest studies of online PPIs failed to find a significant difference in wellbeing between the intervention condition, an exercise intervention, or simply reading a book on positive psychology (Joutsenniemi et al., 2014). However, multiple online studies using waitlist control groups have found significant improvements from PPIs (Drozd et al., 2014; Feicht et al., 2013; Schotanus-Dijkstra et al., 2017). Online interventions that have been effective relative to active control conditions tend to be lower-powered (e.g., $\mathrm{n}<50$ ) and focused on older adults, limiting their generalisability (e.g. Hausmann 
et al., 2017). Thus, larger studies with active control conditions are needed to determine whether MPPIs are more effective than non-MPPI activities.

The current study aimed to address this gap by delivering a six-week online MPPI in a university sample with an active control condition. Three components were chosen for delivery based on past evidence suggesting that between two and four interventions is more effective than one or six interventions (Parks et al., 2012). Given their evidence base and ease of adaptation to an online format, self-compassion, acts of kindness, and positive reminiscence were chosen as interventions for the current study. In comparison, the control group received self-esteem, acts of novelty, and neutral reminiscence modules. The control condition tasks were designed to be as similar as possible to the intervention tasks while omitting the key mechanism believed to account for the positive effects of the interventions. It was hypothesised that there would be a significant interaction between time and condition such that improvements in wellbeing over time would be larger in the treatment group relative to the control group. Changes in self-compassion and depression and anxiety symptoms were also evaluated and expected to improve more in the intervention group relative to controls over the course of the study. A secondary aim of this study was to assess whether baseline levels of resiliency, referring to the level of support systems available to participants, would moderate the effects of the intervention. This was measured using a social-ecological measure developed for adults by the Resilience Research Centre (Liebenberg \& Moore, 2018). It was expected that those with lower levels of resiliency resources at baseline would have more to gain from the intervention and therefore show greater improvements in mental wellbeing than those with higher levels of resiliency resources at baseline.

\section{Materials and Methods}

\subsection{Design}

The study was carried out as a randomized control trial over a six-week period. The intervention and control groups both included three modules, closely matched on activity type. Participants were randomly assigned to a treatment group upon completing the baseline questionnaire using a random number generator in Qualtrics. The order of activities within each condition was also chosen using random number generation with six possibilities. All participants completed the baseline questionnaire prior to gaining access to the modules, which they were instructed to complete once a week over a period of six weeks. This was followed by a questionnaire 7 weeks post-baseline. Participants were not informed which condition they were assigned to until the end of the study.

\subsection{Participants and Procedures}

Participants were first year psychology students from the University of New South Wales who volunteered to participate in the study in exchange for course credit. There were three rounds of data collection over the study period, from June 10th 2019 to April 30th 2020. Round 1 occurred from June 10th to August 11th 2019, Round 2 occurred from September 13th to November 30th 2019, and Round 3 occurred from February 14th to April 30th 2020. For context, Australia's first case of COVID-19 community transmission was reported on March 2nd 2020, and COVID-19 restrictions were gradually introduced from 
mid-March 2020. The University of New South Wales cancelled all in-person classes from March 20th 2020.

Four-hundred twenty-nine participants enrolled in the study, 260 of whom enrolled in Round 3 data collection (i.e., partly during COVID-19). Twenty participants were excluded for not completing the baseline questionnaire. Of the remaining 409 participants, 290 (71\%) were female. The mean age of the sample was $19.61(S D=3.05)$. All consenting participants were eligible to participate. The study was approved by the Human Research Ethics Behavioural Sciences Committee at UNSW (HC3216).

Upon completing the baseline questionnaire, participants were emailed a link to the first weekly activity which was hosted by Qualtrics (Provo, UT). Reminders were emailed five and seven days after the initial email if the task was not yet completed. The link to the weekly tasks was always sent on the same day of the week as the first task. Participants who did not access any of the weekly task links were considered to have withdrawn consent to participate and excluded from data analysis. Of the 409 participants who completed the baseline questionnaires, 326 participants were included in the final analyses once dropouts and non-completers were accounted for.

\subsubsection{Intervention Exercises}

The intervention condition was made up of three modules, each consisting of two weeks of activities (six weeks in total). Participants were randomly assigned the order of modules, given in the format of $\mathrm{ABCABC}$, such that they would complete part one of each module before completing part two of each module in the same order. Full task instructions can be found in the supplementary materials (S1).

2.2.1.1 Self-Compassion The instructions for the self-compassion module were based on descriptions of self-compassion activities described in previous research (Breines \& Chen, 2012). Participants were instructed to consider events that have made them feel negatively about themselves, then imagine how they might respond to a friend going through a similar experience. Compassionate responses were defined based on Neff (2003a) as those focusing on kindness rather than judgement, shared rather than isolating experiences, and balanced rather than polarised responses (not suppressing or exaggerating the consequences). After considering this definition, participants were instructed to write a self-compassionate letter to themselves about those events. Three unique prompts were given in part one and another three unique prompts in part two for participants to consider, for a total of six prompts.

2.2.1.2 Acts of Kindness The acts of kindness instructions were based on those provided in Curry et al. (2018). Participants were asked to brainstorm acts of kindness they would feel comfortable doing for others within the next week. They were specifically instructed to come up with kind acts that they do not usually practice, with either new kind acts for someone they are regularly kind to, or familiar kind acts towards someone they are not usually kind to. After identifying acts of kindness they could complete, participants were instructed to complete the acts before returning to record what they completed. A minimum of $24 \mathrm{~h}$ was required to pass before participants could submit the questionnaire to reduce false responses. In the second part, participants were told they could repeat the same tasks, or different ones. 
2.2.1.3 Positive Reminiscence Positive reminiscence task instructions drew upon research carried out in an online study including young adults (Hallford \& Mellor, 2016). The study reported that positive reminiscence responses that focused on problem solving and identityforming events (i.e., positive events that had a lasting impact on their life) were found to significantly improve self-esteem and meaning, and identity-forming events also improved positive affect. Based on this, three prompts were given in part one and part two that targeted ways in which participants had overcome difficulties in the past or recalled events that had a significant impact on their life. The instructions asked participants to imagine they were telling the story to a friend as they wrote their responses.

2.2.1.4 Control Exercises The control group exercises were chosen based on those used in past research. Self-esteem was used as a control condition for self-compassion (Breines \& Chen, 2012), acts of novelty was used as a control for acts of kindness (Buchanan \& Bardi, 2010), and neutral reminiscence was used as a control for positive reminiscence (Hallford $\&$ Mellor, 2016). The self-esteem module differed from the self-compassion module in that participants were asked to focus on the positive aspects of negative situations in their responses rather than being compassionate to themselves. In the acts of novelty module, participants were asked to come up with ways to change their routine, either by doing activities they do not usually do, or by changing how they go about their usual activities. In the neutral reminiscence module, participants were given prompts about things they were good at or emotional moments instead of those related to overcoming challenging or defining events.

\subsection{Measures}

Mental wellbeing was measured using a composite measure called the COMPAS-W, designed to capture both subjective and psychological aspects of mental wellbeing (Gatt et al., 2014). This questionnaire consists of an overall Wellbeing score in addition to six subcomponents: Composure, relating to one's ability to cope with stressful situations; Own-worth, including self-esteem and autonomy; Mastery, relating to self-confidence and perceived control of one's environment; Positivity, having a positive outlook; Achievement, relating to goal orientation and striving; and Satisfaction, including satisfaction with life, work, health, and relationships. Items are scored on a scale of one to five, from strongly disagree to strongly agree. The measure has been validated in adult and adolescent samples with demonstrated high internal reliability $(r=0.84)$, test-retest reliability $(r=0.82)$ and construct validity (Gatt et al., 2014, 2020). This measure was administered at baseline, week three, week six, and at 7 weeks post-baseline to balance the ability to track progress over the study duration and reducing load on participants.

The 5-item Satisfaction with Life Scale (Diener et al., 1985) was used to measure life satisfaction. This was chosen in addition to the COMPAS-W for its brevity and wide usage as a measure of subjective wellbeing. Items are rated on a 7-point Likert scale. It has high estimated internal reliability in the range of 0.79-0.89, and high test-retest reliability over periods under 4 months (Pavot \& Diener, 2008). This scale was measured weekly.

Stress was measured using the 10-item Perceived Stress Scale (Cohen \& Williamson, 1988; Cohen et al., 1983). It is a well-established stress measure with high internal consistency $($ Cronbach's alpha $=0.89$ ) and established convergent and divergent validity (Roberti et al., 2006). Items are rated on a 5-point scale from 0 indicating Never to 4 indicating Very Often. This scale was administered weekly to monitor ongoing stress levels throughout 
the intervention, particularly because stress was expected to increase during the university semester.

Depression and anxiety symptoms were measured using the 21 -item version of Depression Anxiety Stress Scale (Crawford \& Henry, 2003; Lovibond \& Lovibond, 1995). It is widely used in non-clinical populations to measure negative symptoms. It has been validated in large samples and has demonstrated high test-retest reliability (Crawford \& Henry, 2003). Items are scored on a 4-point scale, with 0 indicating Did not apply to me at all, to 3 indicating Applied to me very much or most of the time. Participants completed this questionnaire at baseline and at 7 weeks post-baseline.

Self-compassion was measured using the Self-Compassion Scale (Neff, 2003b) to determine corresponding changes in self-compassion during the intervention. This questionnaire is the most widely used measure of self-compassion in psychological research. The questionnaire consists of 26 items ranked on a 5-point scale. It includes six subscales: selfkindness, self-judgement, common humanity, isolation, mindfulness, and over-identification. Participants completed this questionnaire at baseline and at 7 weeks post-baseline.

The Resilience Research Centre Adult Resilience Measure (Liebenberg \& Moore, 2018) was measured at baseline as an indicator of resiliency resources. It includes 28 items with a 5-point response scale ranging from Describes me extremely well to Does not describe me. The scale is designed to measure the contextual resources that support resilience processes. These include aspects of social and community inclusion, family support, spirituality, identity, and personal skills and competencies.

\subsection{Data Analysis}

\subsubsection{Power Analysis}

An a priori power analysis was conducted on the basis of a repeated-measures, within by between interaction model using $G^{*}$ power3 (Faul et al., 2007). The power analysis was conducted on the basis of a small effect size $(f=0.10)$, with $\alpha=0.05$ and only two time points to ensure power for measures taken only at baseline and 7 weeks post-baseline. The results indicated a sample of 200 participants $(n=100)$ would be required for $80 \%$ power, and a sample of $266(n=133)$ participants would be required for $90 \%$ power. Due to higher than expected attrition, the final analysis was instead conducted using a linear mixed model which is better able to handle missing data (Krueger \& Tian, 2004).

\subsubsection{Missing Data}

Multiple imputation was conducted using the mice package in $R$ (R Core Team, 2020; van Buuren \& Groothuis-Oudshoorn, 2011). This was done separately for each condition using the same method involving classification and regression trees (cart method). Twenty-five imputations were run based on the overall percentage of data missing, which was approximately $25 \%$ of all data. 


\subsubsection{Modelling}

Linear mixed models were conducted in $R$ with the lme 4 package (Bates et al., 2015) using all 25 data imputations and the original, non-imputed dataset. Results from each imputation were pooled using mice (van Buuren \& Groothuis-Oudshoorn, 2011). Participant ID was added as the random effect and mental health outcomes as fixed effects. We then tested whether the effects were dependent on baseline resiliency resources or gender by adding interaction terms with each variable to each model. Significant resiliency moderation effects were explored post hoc by dividing the sample into three equal groups and testing the time by group interaction within each level of resiliency resources. This method was also used to plot the three-way interactions. To evaluate effect size, while Cohen's $d$ is not generally reported as an effect size for linear mixed models due to its inability to capture the covariance structure of the random effects, it was estimated here for the significant intervention effects to provide a more direct comparison to past findings. Cohen's $d$ was estimated from the standardised beta coefficients and standard error using the following formula: $\beta / \sqrt{ }(n)^{*}$ SE. Cohen's $d$ cannot be estimated for interactions including continuous measures as it is designed for group-based comparisons only. As a rule of thumb, Cohen's $d$ effects sizes of 0.20 are considered 'small', 0.5 are considered 'medium', and 0.8 are considered 'large' effects.

As the study was partly conducted during 2020 when Australia began to introduce social distancing restrictions to reduce the spread of COVID-19, we also tested whether participating during these restrictions had an impact on the efficacy of the intervention. To account for this, additional models were run with a continuous variable representing the number of weeks that participants were enrolled in the study during COVID-19 restrictions in Australia (entered as March 13, 2020). Thus, participants who enrolled on or after March 13 would have a score of seven indicating they would have experienced seven weeks of total COVID-19 restrictions by the end of their study participation, whereas participants who were enrolled on February 14, 2020 (the first day of Round 3) would have a score of three and those who enrolled in Round 1 and 2 were given a score of zero.

\section{Results}

\subsection{Participant Characteristics}

Of the 429 participants who enrolled in the study, 20 were excluded because they failed to complete the baseline questionnaire, and a further 83 were excluded because they withdrew from the study or did not access any of the weekly tasks. The remaining sample $(N=326$, $n=163)$ had a mean age of $19.7(S D=3.2)$ and included 229 females (70\%). One hundred and twenty-five participants completed all six of the weekly activities. A CONSORT diagram depicting participant flow is shown in Fig. 1.

As shown in Table 1, no significant differences were found at baseline for any of the outcome variables of interest including wellbeing, life satisfaction, stress, depression and anxiety symptoms, self-compassion, nor resiliency resources between the control group and the intervention group. T-tests comparing participants who completed at least five of the weekly activities to those who completed less than five suggested that completers had significantly higher Satisfaction with Life Scale scores $(M=24.97, S D=6.57$ vs $M=22.93, \mathrm{SD}=6.57)$, lower Perceived Stress Scale scores $(M=16.47, S D=6.48$ 


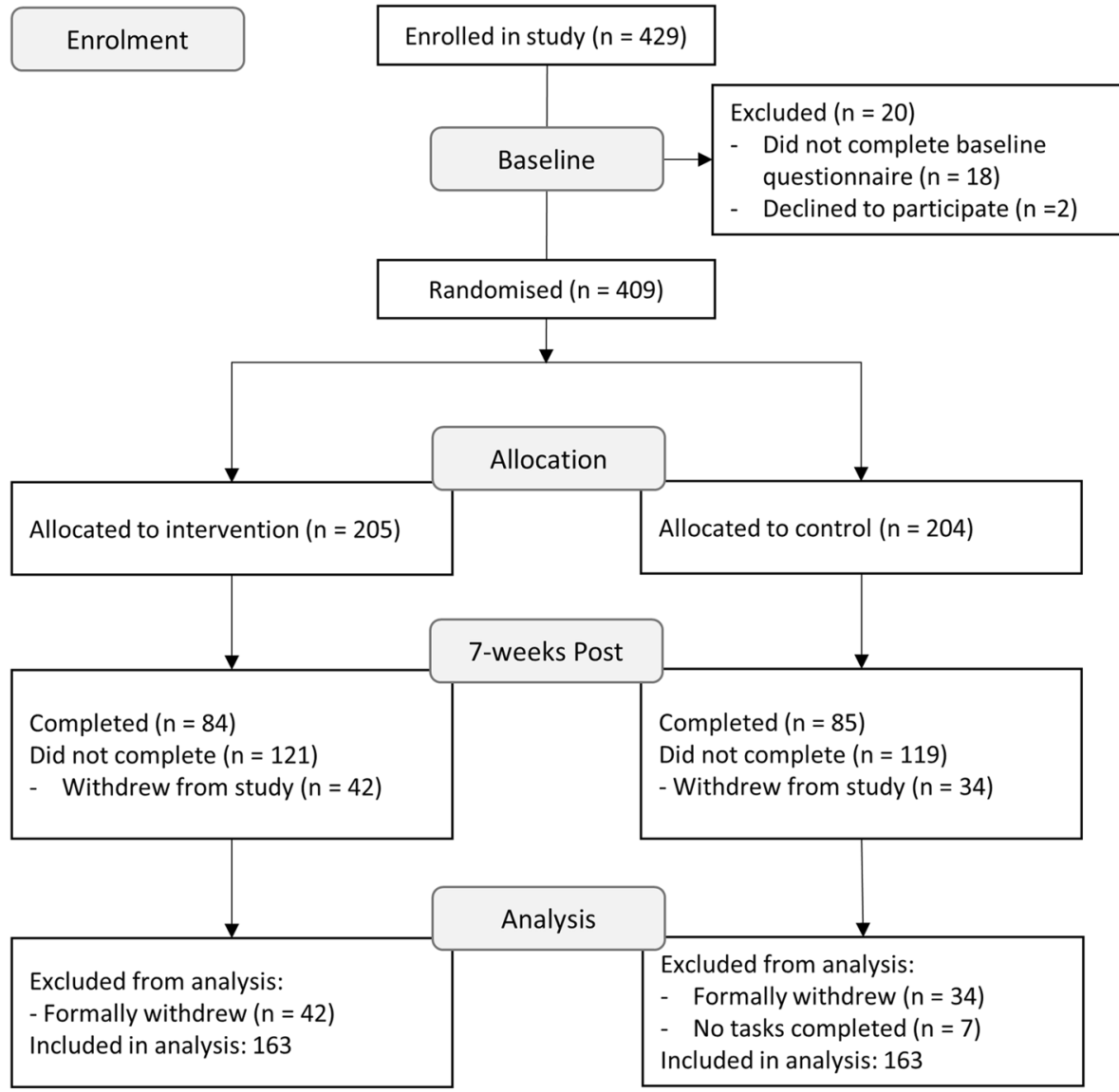

\section{Weekly participation breakdown}

\begin{tabular}{llcccccc}
\hline Condition & & Week 1 & Week 2 & Week 3 & Week 4 & Week 5 & Week 6 \\
Intervention & Task and survey & 131 & 109 & 95 & 87 & 89 & 84 \\
& Survey only & 32 & 28 & 24 & 13 & 4 & 11 \\
& Not completed & 42 & 68 & 86 & 105 & 112 & 110 \\
\multirow{4}{*}{ Control } & Task and survey & 132 & 125 & 128 & 93 & 89 & 89 \\
& Survey only & 31 & 20 & 14 & 15 & 18 & 5 \\
& Not completed & 41 & 59 & 62 & 96 & 97 & 110 \\
\hline
\end{tabular}

Fig. 1 CONSORT diagram of study participation. Notes. Task and survey indicates full completion for that week; Survey only indicates that the outcome measures were completed, but the intervention/control task was not completed

vs $M=18.78, S D=6.36)$, and lower DASS-21 total scores $(M=25.57, S D=20.50$ vs $M=30.93, S D=23.01$ ) than non-completers. 
Table 1 Descriptive statistics and t-tests comparing the intervention with the control group at baseline

\begin{tabular}{|c|c|c|c|c|c|c|}
\hline \multirow[t]{3}{*}{ Variable } & \multirow{2}{*}{\multicolumn{2}{|c|}{$\begin{array}{l}\text { Intervention } \\
n=163\end{array}$}} & \multirow{2}{*}{\multicolumn{2}{|c|}{$\begin{array}{l}\text { Control } \\
n=163\end{array}$}} & \multirow[t]{3}{*}{$t(324)$} & \multirow[t]{3}{*}{$p$ value } \\
\hline & & & & & & \\
\hline & $M$ & $S D$ & $M$ & $S D$ & & \\
\hline SWLS & 23.55 & 6.89 & 23.88 & 6.38 & 0.45 & 0.65 \\
\hline PSS & 18.56 & 6.52 & 17.23 & 6.42 & 1.84 & 0.07 \\
\hline COMPAS-W & 94.29 & 12.77 & 94.97 & 12.46 & 0.49 & 0.63 \\
\hline DASS- $21^{+}$ & 3.10 & 0.94 & 2.99 & 1.02 & 1.00 & 0.32 \\
\hline SCS & 2.99 & 0.63 & 2.94 & 0.56 & 0.68 & 0.50 \\
\hline RRC-ARM & 107.66 & 16.53 & 106.16 & 17.51 & 0.79 & 0.43 \\
\hline
\end{tabular}

SWLS Satisfaction with life scale, PSS Perceived stress scale, COM$P A S-W$ COMPAS-W wellbeing score, DASS-21 Depression anxiety stress scale, $S C S$ Self-compassion scale, $R R C$-ARM Resilience Research Centre adult resilience measure

${ }^{+}$DASS-21 scores were log-transformed

Bivariate Pearson correlation coefficients between key measures at baseline are shown in Table 2. All correlations were significant. As expected, mental wellbeing, life satisfaction, self-compassion, and resiliency resources were all positively correlated with one another, including COMPAS-W subscales, and each was negatively correlated with perceived stress and depression and anxiety symptoms.

\subsection{Intervention Effects}

\subsubsection{COMPAS-W Wellbeing}

There was a significant main effect of time for mental wellbeing, indicating that when averaged across both conditions, there was a reduction in wellbeing from baseline to week three $(\beta=-0.15, t=-2.52, p=0.012)$ and week six $(\beta=-0.14, t=-2.37, p=0.018)$ that was not maintained through to 7 weeks post-baseline $(p=0.465)$. There was no significant interaction between condition and time. However, the moderation analyses showed that the treatment effect on wellbeing was dependent on whether students participated during COVID-19 restrictions and their baseline resiliency resources. While there was no observed effect of COVID-19 restrictions on wellbeing, accounting for this in the model revealed a significant condition by time interaction at 7 weeks post-baseline $(\beta=0.33$, $t=2.21$, estimated Cohen's $d=0.17, p=0.028$ ), indicating greater improvements in wellbeing in the intervention group relative to the active control group from baseline to 7 weeks post-baseline (Fig. 2a, Table S1). There was also a significant three-way interaction between resiliency resources, time, and condition at week three $(\beta=-0.15, t=-1.36$, $p=0.054)$ and week six $(\beta=-0.19, t=-2.32, p=0.021)$, indicating greater improvements in wellbeing for those with lower levels of baseline resiliency resources. To examine this effect further, the time by condition interaction was tested in participants with high, medium, and low resiliency resources based on tertile cut-offs. We found that the low baseline resiliency group showed greater improvement in the intervention relative to the control condition at week six $(\beta=0.34, t=2.24$, estimated $d=0.18, p=0.026)$ and 7 weeks postbaseline $(\beta=0.35, t=2.31$, estimated $d=0.18, p=0.022)$ (Fig. 3a, Table S1). There was a 


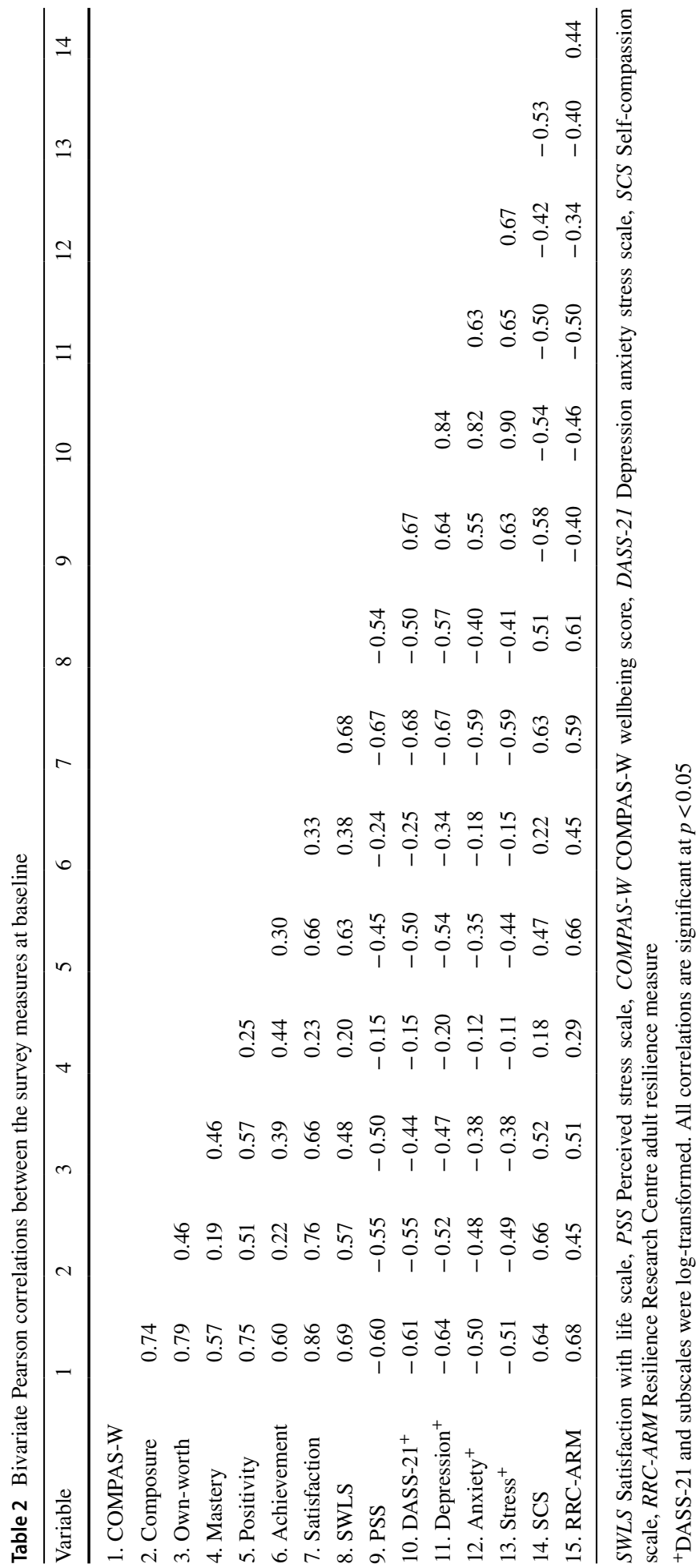



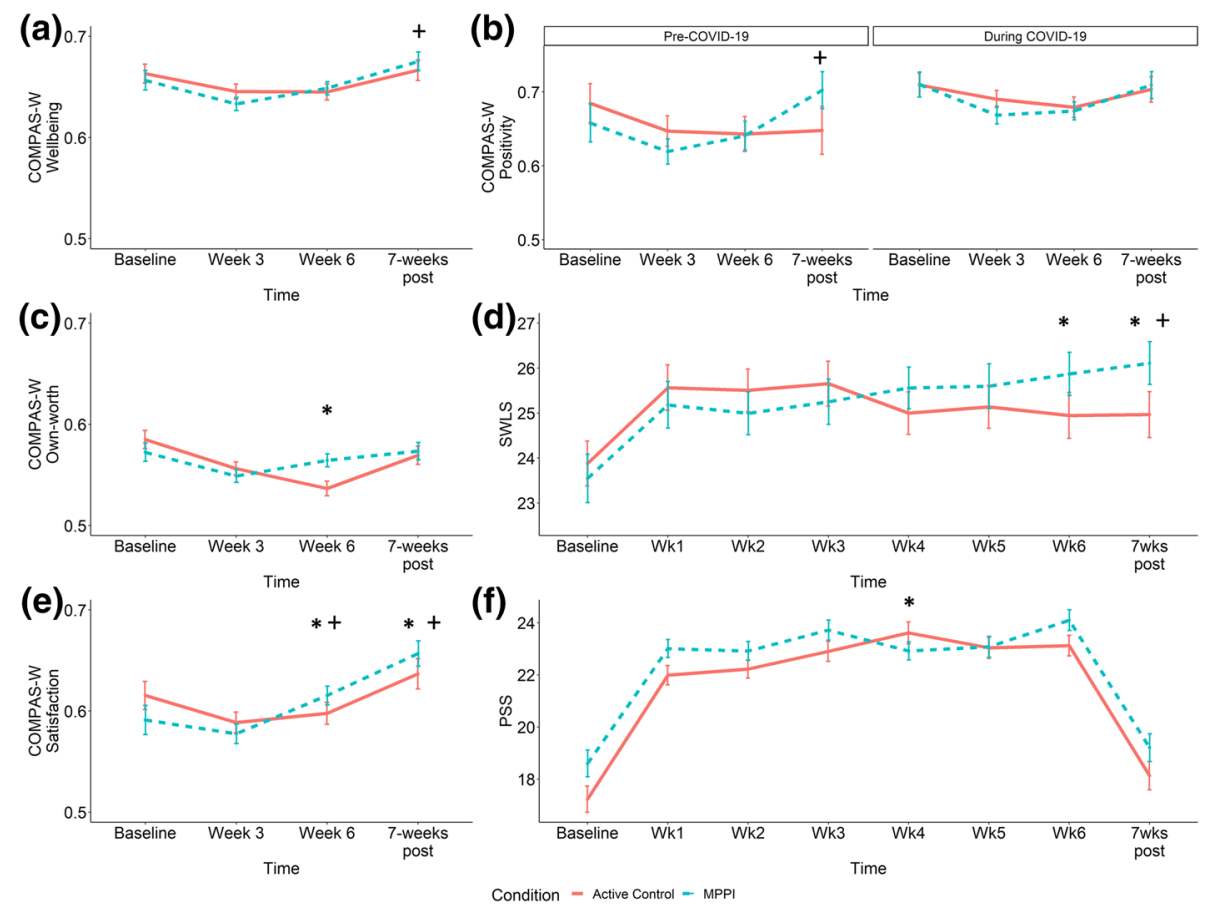

Fig. 2 Signficant overall effects of the intervention relative to the active control group for a COMPAS-W Wellbeing, b COMPAS-W Positivity (moderated by COVID-19 restrictions), c COMPAS-W Own-worth, d Satisfaction with Life Scale (SWLS), e COMPAS-W Satisfaction, and f Perceived Stress Scale (PSS) scores. *Indicates significantly larger improvements in the intervention group compared to the control group relative to baseline without accounting for COVID-19 restrictions. + Indicates significantly larger improvements in the intervention group compared to the control group relative to baseline when accounting for COVID-19 restrictions. COMPAS-W scores have been normalised to a scale of 0-1 for comparative purposes

strong overall relationship between resiliency and wellbeing, indicating that higher resiliency was associated with higher wellbeing throughout the intervention.

\subsubsection{COMPAS-W Composure}

Composure significantly increased from baseline to week six $(\beta=0.14, t=2.00, p=0.046)$ and 7 weeks post-baseline $(\beta=0.16, t=2.29, p=0.023)$ when averaging across both conditions (Table S2). Neither of these effects remained when accounting for COVID-19 restrictions. There were no effects of condition, and effectiveness of the intervention was not moderated by baseline resiliency resources or gender.

\subsubsection{COMPAS-W Own-Worth}

Own-worth significantly reduced from baseline to week three $(\beta=-0.26, t=-2.85$, $p=0.004)$ and week six $(\beta=-0.44, t=-4.64, p<0.001)$, but this was not maintained at post $(p=0.197)$. A significant condition by time interaction was found for Own-worth at week six $(\beta=0.38, t=2.80$, estimated $d=0.22, p=0.005)$, with the control condition 

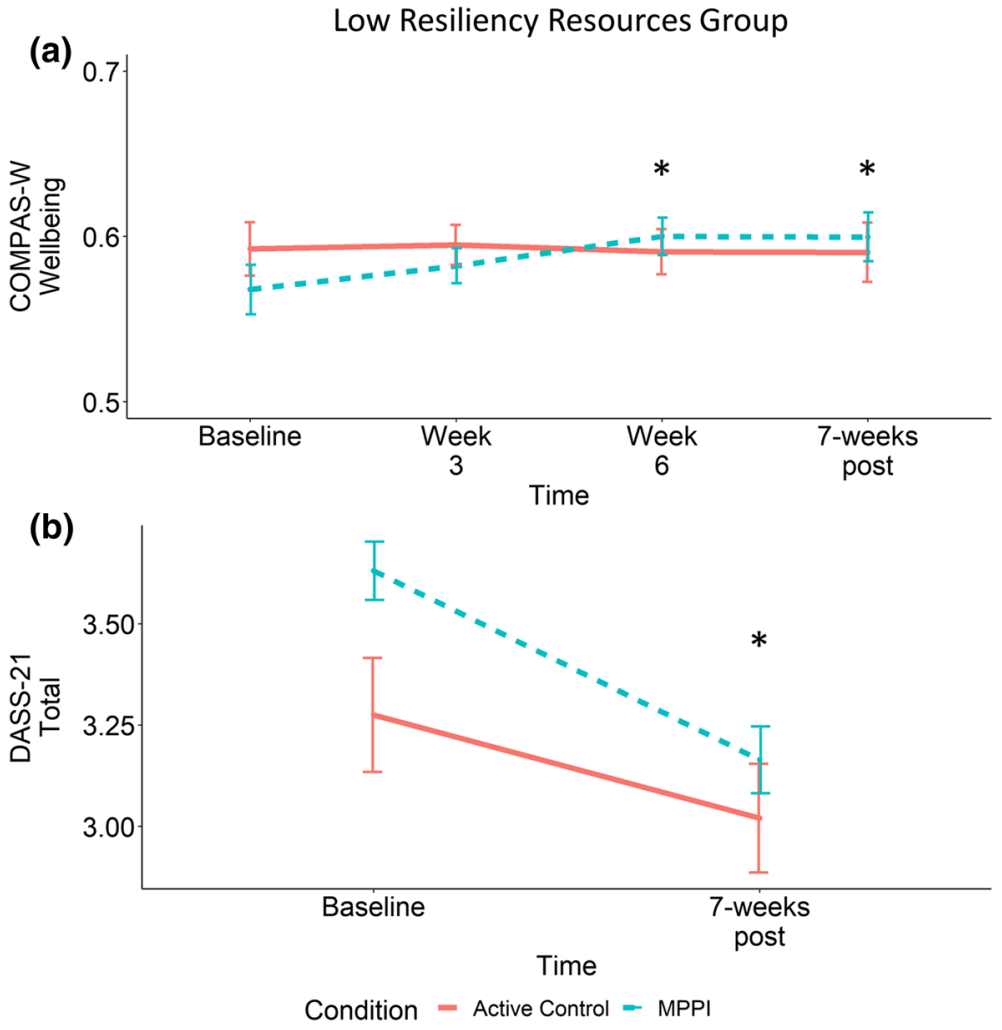

Fig. 3 Significant moderation of intervention effects at low baseline resiliency resources for a COMPAS-W Wellbeing and b log-transformed DASS-21 depression and anxiety total scores. Low resiliency was defined as a score less than $101(\mathrm{~N}=104, \mathrm{n}=54$ in Intervention group) using the Resilience Resource Centre Adult Resilience Measure (RRC-ARM). *Indicates significantly greater improvements in the intervention group compared to the control group relative to baseline. COMPAS-W scores were normalised to a scale of $0-1$ for comparative purposes

showing a reduction in own-worth over time as compared to the intervention group who instead showed little change over time (Fig. 2c, Table S3). When accounting for COVID19 restrictions, the main effect of time on Own-worth at week three $(p=0.211)$ and the interaction effect at week six was no longer significant $(p=0.069)$, but there was still a significant main effect from baseline to week six showing a reduction in Own-worth averaged across both groups $(p=0.004)$. The effect of the intervention was not moderated by baseline resiliency resources or gender.

\subsubsection{COMPAS-W Mastery}

There was a significant reduction in Mastery from baseline to week three $(\beta=-0.25$, $t=-3.00, p=0.003)$ and week six $(\beta=-0.26, t=-3.05, p=0.002)$ when averaging across treatment and control conditions that was not maintained through to 7 weeks postbaseline ( $p=0.205$, Table S4). These effects remained when accounting for COVID-19 restrictions. There was no significant time by condition interaction, but there was a three way-interaction between time, condition, and resiliency resources such that the intervention 
was more effective relative to the control condition for individuals with lower baseline resiliency resources. However, when participants were split into high, medium, and low resiliency groups, no group showed significantly greater improvements in the intervention condition relative to controls over time. The effect of the intervention was not moderated by gender.

\subsubsection{COMPAS-W Positivity}

There was a significant decrease in Positivity from baseline to week three $(\beta=-0.14$, $t=-2.09, p=0.037)$ and week six $(\beta=-0.19, t=-2.73, p=0.007)$ that were not maintained through to 7 weeks post-baseline $(p=0.286)$. However, when accounting for COVID-19 restrictions, the reductions in Positivity at week three $(p=0.144)$ and week six $(p=0.062)$ were not significant. Accounting for COVID-19 restrictions revealed a significant time by condition interaction at 7 weeks post-baseline demonstrating greater improvements in Positivity for the intervention group relative to the control group $(\beta=0.49$, $t=2.79$, estimated $d=0.21, p=0.006$ ). There was also a three-way interaction between time, condition, and COVID-19 at 7 weeks post-baseline $(\beta=-0.12, t=-2.40, p=0.017)$ showing that improvements in Positivity for the intervention group relative to the control group were smaller for individuals participating during COVID-19 restrictions compared to those who participated before the restrictions were introduced (Fig. 2b, Table S5). Inspection of the graphs suggested the reduced efficacy of the intervention during COVID-19 could be due to the participants in Round 3 having higher baseline Positivity than participants in Rounds 1 and 2. A t-test revealed that the difference in baseline Positivity between rounds was marginally significant $(t(169)=1.52, p=0.085)$. The effect of the intervention was not moderated by baseline resiliency resources or gender.

\subsubsection{COMPAS-W Achievement}

There was no evidence for any effect of the intervention or time on Achievement in either the control or intervention conditions over the study period, and no moderating effect of COVID-19, baseline resiliency, or gender (Table S6).

\subsubsection{COMPAS-W Satisfaction}

There was a significant decrease in Satisfaction from baseline to week three when averaging across both groups $(\beta=-0.16, t=-2.39, p=0.017)$. However, this effect was not maintained at week six $(p=0.151)$ and reversed with a significant positive effect of time by 7 weeks post-baseline $(\beta=0.14, t=2.05, p=0.041)$. There was a significant time by condition interaction indicating greater improvements in Satisfaction for the intervention compared to controls at week six $(\beta=0.25, t=2.53$, estimated $d=0.19, p=0.012)$ and 7 weeks post-baseline $(\beta=0.29, t=2.90$, estimated $d=0.23, p=0.004)$ relative to baseline (Fig. 2e, Table S7). Participating during COVID-19 restrictions was found to have a somewhat positive effect on Satisfaction $(\beta=0.07, t=2.02, p=0.043)$, and the intervention effect found at week six $(\beta=0.41, t=2.59$, estimated $d=0.20, p=0.010)$ and 7 weeks post-baseline remained significant $(\beta=0.48, t=2.83$, estimated $d=0.22, p=0.005)$ (Fig. 2e, Table S7). Furthermore, the main effects of time on Satisfaction at week three $(p=0.116)$ and 7 weeks post-baseline $(p=0.766)$ were not significant when accounting for COVID-19 restrictions 
in the model. The impact of the intervention on Satisfaction was not moderated by baseline resiliency resources or gender.

\subsubsection{Satisfaction with Life Scale (SWLS)}

There was a significant increase in life satisfaction each week of the program relative to baseline, averaging across both conditions $(\beta=0.18, t=3.41, p=0.001$ at 7 weeks postbaseline). There was a significant time by condition interaction indicating greater improvement in life satisfaction for the intervention group relative to the control group from baseline to week six $(\beta=0.18, t=2.47$, estimated $d=0.20, p=0.014)$ and 7 weeks post-baseline $(\beta=0.23, t=3.07$, estimated $d=0.26, p=0.002)$ (Fig. 2d, Table S8). When accounting for COVID-19 restrictions, the main and interaction effects at 7 weeks post-baseline remained significant $(\beta=0.29, t=2.48$, estimated $d=0.19, p=0.014)$, but the interaction effect at week six did not $(p=0.096)$ (Fig. $2 d$, Table S8). There was also a significant positive main effect of participating during COVID-19 restrictions on life satisfaction $(\beta=0.09$, $t=2.35, p=0.019)$. The effect of the intervention was not moderated by baseline resiliency resources or gender.

\subsubsection{Perceived Stress Scale (PSS)}

There was a significant main effect of time on perceived stress such that when averaged across both groups, stress was higher during weeks one to six relative to baseline. There was a significant time by condition interaction at week four indicating lower perceived stress for the intervention condition relative to the control condition $(\beta=-0.31, t=-2.97$, estimated $d=-0.24, p=0.003$ ) (Fig. 2f, Table S9). A similar trend was evident at week five but did not reach significance $(\beta=-0.19, t=-1.79, p=0.073)$. These interactions were not significant when accounting for COVID-19 restrictions $(p<0.279)$.

The main effect of time on perceived stress was moderated by baseline resiliency, indicating that those with more resiliency resources at baseline had larger increases in stress during the program, averaged across conditions up to week five $(\beta=0.14, t=2.63$, $p=0.009)$. There was also a main effect of gender such that male participants had lower stress than females at week one $(\beta=-0.50, t=-3.33, p=0.001)$, and there was a significant time by gender interaction indicating that males had larger increases in stress at week three compared to females $(\beta=-0.30, t=-2.03, p=0.042)$.

\subsubsection{Depression, Anxiety, Stress Scale (DASS-21)}

A significant main effect of time was found for the DASS-21 scores, indicating a reduction in symptoms over time when averaged across both groups $(\beta=-0.23, t=-3.20$, $p=0.001)$. There was no interaction between condition and time, showing no difference between the intervention and control condition at the end of the program. When accounting for COVID-19 restrictions, the main effect of time on DASS-21 scores was no longer significant $(p=0.248$ ), and there was a main effect of condition showing that the intervention group had lower DASS-21 scores overall relative to the control group, but no interaction suggests the change over time was similar between the two groups. The effect of the intervention was moderated by baseline resiliency $(\beta=0.21, t=2.10, p=0.036)$, indicating that the intervention group showed greater symptom reductions relative to the control group for participants with low baseline resiliency resources. This effect is shown in 
Fig. 3b (Table S10). The effect of the intervention was not moderated by gender. An analysis of the DASS-21 subscales revealed that the main effect on total DASS-21 scores was driven by reductions in anxiety $(\beta=-0.41, t=-5.29, p<0.001)$ and stress $(\beta=-0.25$, $t=-3.53, p=0.001) 7$ weeks post-baseline, but not by change in depressive symptoms (Tables S11-S13). However, none of the subscales were significantly moderated by resiliency as was observed for the total scale (Tables S11-S13).

\subsubsection{Self-Compassion Scale}

No change in self-compassion was noted throughout the experiment, and no interactions were found with COVID-19 restrictions, gender, or baseline resiliency (Table S14).

\section{Discussion}

The aim of this study was to test the effectiveness of a six-week positive psychology intervention program against an active control group. The results indicate that an intervention made up of self-compassion, acts of kindness, and positive reminiscence can improve life satisfaction to a greater degree than comparable control tasks. This was found with both the COMPAS-W Satisfaction subscale $(\beta=0.29$, estimated $d=0.22)$ and the Satisfaction with Life Scale $(\beta=0.23$, estimated $d=0.25)$, with both effects emerging regardless of COVID-19 restrictions. COMPAS-W Own-worth (measuring autonomy and self-esteem) also improved in the intervention relative to the control group at week six compared to baseline but was not significant when accounting for COVID-19 restrictions. In contrast, greater improvements in composite wellbeing and COMPAS-W Positivity were found for the intervention group relative to the control group only when accounting for COVID-19 restrictions. The effect of the intervention on composite wellbeing and DASS-21 scores was moderated by baseline resiliency such that those with poor resiliency resources at the start of the trial benefitted more from the intervention condition than those with more resources. Thus, the current trial demonstrates that MPPIs delivered online can be effective in improving life satisfaction regardless of resiliency resources and may improve composite wellbeing and reduce depression, anxiety, and stress symptoms in individuals with poor perceived support.

The positive effect of the intervention on subjective wellbeing indicated by improvements in life satisfaction (measured here using both the COMPAS-W Satisfaction subscale and the SWLS) and positivity (COMPAS-W Positivity) is consistent with past studies which have found online MPPIs to improve subjective wellbeing relative to waitlist control groups (Drozd et al., 2014; Feicht et al., 2013; Schotanus-Dijkstra et al., 2017). This improvement has been found across a range of subjective wellbeing measures, including the WHO five-factor wellbeing index after a seven-week intervention (Feicht et al., 2013), affect balance after a four-week intervention (Drozd et al., 2014), and all components of the Keyes' Mental Health Continuum (Short Form) in those who completed a supported self-help program over eight to twelve weeks (Schotanus-Dijkstra et al., 2017). The effect sizes reported in the current study for measures of subjective wellbeing were small, ranging from $d=0.22$ to $d=0.25$ overall (or $0.19-0.22$ when accounting for COVID). While lower than the overall effect size reported for MPPIs in a recent meta-analysis $(g=0.34$ for subjective wellbeing), our effect sizes are similar to the effect size reported when lowquality studies, defined as meeting less than three of six criteria outlined by the Cochrane 
Collaboration assessment tool (Higgins et al., 2011), were excluded from the analysis $(g=0.26)$ (Hendriks et al., 2020). The current study therefore provides evidence that online MPPI programs as short as six weeks are a viable approach to improving subjective wellbeing over and above any placebo effect.

In contrast to the effects found for subjective wellbeing, the current intervention was not as effective in improving measures of psychological wellbeing (e.g., COMPAS-W Own-worth, Mastery and Achievement). Other than the study by Schotanus-Dijkstra et al. (2017), very few studies have examined composite or psychological measures of wellbeing in relation to online MPPIs. The current findings suggest that while composite or psychological wellbeing can be improved (e.g., for both composite wellbeing and Own-worth), an online intervention of six weeks is not sufficient to produce robust effects. Most interventions that have shown improvements in psychological wellbeing in particular used longer trial duration of nine to twelve weeks and are most often delivered in person (Hendriks et al., 2020), which could explain why the improvements in composite wellbeing and Ownworth reported here were only weak effects $(d=0.17$ and 0.23 , respectively) in comparison to past studies $(g=0.31)$. Furthermore, while improvements in psychological wellbeing have been reported previously for online interventions, this was in the context of participants having email support from a personal counsellor in addition to the assigned activities, which likely improved the effectiveness of the intervention compared the current study where all activities were completely self-administered (Schotanus-Dijkstra et al., 2017). Nonetheless, the current study found evidence for temporary benefits in Ownworth $(d=0.23)$, capturing psychological wellbeing domains relating to self-esteem and autonomy, and improvements in composite wellbeing were apparent for individuals with poor baseline resiliency. A longer intervention period may improve the effectiveness of the interventions used here. It is also possible that the specific intervention tasks applied here are better suited to improving subjective components of wellbeing. Future research should explore whether including activities more targeted to psychological wellbeing outcomes would translate to broader effects. Such strategies may include activities that focus on promoting elements of Own-worth, Mastery and Achievement (e.g., activities focusing on building self-confidence, strengths, and goal striving).

In addition to effects on wellbeing, there is some evidence that the intervention impacted depression and anxiety symptoms measured using the DASS-21. Specifically, only intervention participants with poor baseline resiliency showed greater reductions in overall scores compared to control participants. This effect was significant only for the total DASS-21 score and not the subscales. By comparison, the Perceived Stress Scale (PSS) was not moderated by resiliency, and the significant reduction in perceived stress for the intervention relative to the control group at week four did not remain significant when accounting for COVID-19 restrictions. It is notable however that the DASS-21 and PSS are two different measures of distress. While the DASS-21 measures symptoms of depression, anxiety and stress including for example an inability to relax or a feeling of tension (Lovibond \& Lovibond, 1995), the Perceived Stress Scale measures the appraisal of events as more or less stressful (Cohen \& Williamson, 1988). Thus, the intervention appears to have been more useful in improving how some symptoms of depression and anxiety are dealt with despite life events (like COVID-19) still being appraised as stressful. Future trials could implement activities that also focus on the cognitive appraisal of stressful life events, such as cognitive behavioural therapy.

It is important to consider the co-occurrence of the COVID-19 pandemic during the final data collection phase of this study. More than half of the participants were undergoing the study while COVID-19 restrictions were introduced in Australia in March and 
April 2020. Accounting for COVID-19 restrictions had an impact on some of the study outcomes, particularly in relation to the COMPAS-W total Wellbeing score, where a significant effect of the intervention relative to the control condition was only significant when COVID-19 restrictions were accounted for. Participating during COVID-19 restrictions may have impacted the effectiveness of the Acts of Kindness intervention in particular as participants were more limited in the types of social interactions they could partake in during this time. We also found a three-way interaction between time, condition, and COVID19 restrictions for COMPAS-W Positivity indicating that while the intervention group showed significant improvements in Positivity relative to the control group $(d=0.21)$, these improvements were smaller for those who participated during COVID-19 restrictions. While this could be interpreted as a negative effect of the pandemic, observation of Fig. $2 \mathrm{~b}$ suggests that it might be explained by the higher baseline Positivity observed in Round 3 compared to earlier rounds of data collection. This was supported by a t-test that showed a marginally significant trend in this direction $(p=0.085)$. This difference could be explained by the timing of Round 3 data collection relative to the academic year. That is, these participants were first year students participating at the beginning of their university training after their summer and Christmas break. In comparison, participants who did not participate during COVID-19 restrictions were in the middle or end of their academic year, which is when mental health problems are known to increase (Duffy et al., 2020). Thus, the reduced effectiveness of the intervention in improving Positivity during COVID-19 restrictions might actually be explained by these participants having higher Positivity from the beginning of the intervention, resulting in a ceiling effect. Due to this potential confound, COVID-19 effects should be interpreted with caution.

The current study was designed to examine whether a combination of PPIs can improve mental wellbeing rather than to test whether specific interventions were effective. Therefore we cannot conclude whether some activities or certain prompts contributed more to the overall effects than others. For instance, while the intervention overall showed significant improvements in wellbeing, there was no significant improvement in self-compassion scores despite the expectation that improved self-compassion would mediate improvements in mental wellbeing (Zessin et al., 2015). It is therefore unclear whether this null result reflects an ineffective self-compassion exercise or a measurement of self-compassion not sensitive to intervention changes. Also, because self-compassion was only measured at the beginning and end of the program, it is possible that the intervention produced temporary improvements in self-compassion scores that were not measured at intermediary timepoints and not maintained through to the final assessment. Furthermore, the salience of the event that participants recalled for the self-compassion activity may have varied between participants, potentially affecting its impact. Yet, the salience of events reported was not examined here so we cannot determine their impact. In future studies, alternative and more frequent measures of self-compassion and the salience of chosen events would be needed to clarify these effects.

The use of an active control group instead of a waitlist control is a key strength of the current study. This experimental design helped keep attrition rates similar in both groups as the amount of time spent on tasks was comparable. It also provided evidence that MPPI activities are indeed more effective than non-MPPI activities and helps rule out a placebo effect as participants were blinded to their assigned condition until the conclusion of the study. These results are in contrast to a number of past online MPPIs that did not find significant improvements in mental wellbeing relative to active controls. For instance, in a large community sample of Finnish adults, an intervention consisting of good deeds, gratitude, optimism, and reducing rumination produced no significant improvement in 
happiness as measured by the Happiness-Flourishing Scale, or depressive symptoms in the intervention group relative to controls who either exercised or read a book about happiness (Joutsenniemi et al., 2014). However, they did find that the good deeds intervention resulted in the greatest improvements in happiness relative to the other activities, indicating some evidence in support of this specific PPI. Another study conducted in HIV-positive patients failed to find significant improvements in positive affect after a five-session intervention when compared with facilitator interviews (Moskowitz et al., 2017). In contrast, one small study of older adults with osteoarthritis found a significant improvement in life satisfaction and reduction in negative affect following a six-week phone program compared to an active control group (Hausmann et al., 2017). While the current study was based on a very specific age group and socio-educational demographic of university students, it was a relatively larger sample than many previous studies and showed further evidence that online MPPIs can effectively improve subjective wellbeing relative to active controls in a different age group. While current and past findings suggests that MPPIs can be effective across the lifespan, further work should assess whether the findings generalise to different socio-economic backgrounds and vulnerable groups.

Apart from the specific socio-demographic targeted in the current sample, another limitation of the current study is the significant amount of attrition that occurred over the intervention period. This was handled using multiple imputation to account for uncertainty in the missing the data, using an intention to treat analysis, and a larger sample was recruited over three rounds to obtain more study completers. However, it is impossible to know whether individuals who did not complete the study would have responded differently from those who remained, especially given that those who continued with the study had higher life satisfaction, lower stress and DASS-21 scores at baseline relative to non-completers. Given that we found greater improvements in those with lower resiliency at baseline, it is possible that the intervention effects may have been even more apparent if the non-completers remained in the program. However, it is also possible that those who withdrew from the study did so due to finding the intervention ineffective in meeting their needs. Moreover, while our study was conducted completely online, other approaches using in-person assessments showed a much lower attrition rate (16\%) and have suggested that taking this approach could help lower attrition rates in future research (Kushlev et al., 2020). Another limitation is the potential homogeneity within the three training activities that might arise from differences in the salience of events selected (e.g., for the self-compassion or reminiscence exercises), and differences in existing social networks (e.g., for the acts of kindness). In the current study, we did not collate measures of salience or personal networks, but instead analysed the impact of all three training modules as a whole. Future studies could consider such variations and how they may potentially modify the outcomes. Finally, our last time-point was 7 weeks post-baseline and so we were unable to examine the long-term maintenance of any significant effects found. Studies using similar intervention tasks have found effects lasting up to six months (Hausmann et al., 2017) suggesting that long-term effects may be present. This remains to be confirmed in future studies.

In conclusion, this study provides new evidence of the effectiveness of MPPIs relative to active control activities in primarily improving subjective wellbeing. The intervention remained effective even when delivered under COVID-19 restrictions in Australia, and there is evidence that composite wellbeing improved more in the intervention group when the impact of COVID-19 restrictions was accounted for in the model. The intervention was found to be more effective in improving composite wellbeing, and depression and anxiety symptoms within individuals with low baseline resiliency resources, suggesting that MPPIs may be particularly useful for those with fewer resources to cope with stressful situations. 
Future studies are needed to determine whether different MPPI activities could be used to promote improvements in other aspects of wellbeing such as the COMPAS-W subscales of Composure, Mastery and Achievement. In summary, the current study indicates that online MPPIs including acts of kindness, positive reminiscence, and self-compassion outperform active control tasks and show promise as a means to improve mental wellbeing and symptoms of distress.

Supplementary Information The online version contains supplementary material available at https://doi. org/10.1007/s10902-021-00449-3.

Acknowledgements The authors would like to thank Madeleine Rhodes for her assistance in data collection, and Peter Humburg for his statistical guidance.

Author contributions MRC conceptualised the study, created the materials, carried out recruitment \& data collection, analysis, and wrote the first draft of the paper. JMG helped guide study design, research questions for analysis and interpretation, and edited drafts.

Funding MRC was supported by the Australian Government Research Training Scholarship and the Neuroscience Research Australia top-up scholarship. JMG was supported by a National Health and Medical Research Council (NHMRC) Project Grant (1122816).

Data and code availability Data and code used to produce the results will be made available upon reasonable request.

\section{Declarations}

Conflict of interest MRC has no conflict of interest. JMG holds stock in MAP Biotech Pty Ltd.

Ethics approval The study was approved by the Human Research Ethics Behavioural Sciences Committee at UNSW (HC3216).

Informed consent All participants provided informed consent prior to participating.

\section{References}

Bao, K. J., \& Lyubomirsky, S. (2014). Making happiness last: Using the hedonic adaptation prevention model to extend the success of positive interventions. The Wiley Blackwell Handbook of Positive Psychological Interventions. https://doi.org/10.1002/9781118315927.ch21

Bates, D., Mächler, M., Bolker, B. M., \& Walker, S. C. (2015). Fitting linear mixed-effects models using lme4. Journal of Statistical Software. https://doi.org/10.18637/jss.v067.i01

Bolier, L., Haverman, M., Westerhof, G. J., Riper, H., Smit, F., \& Bohlmeijer, E. (2013). Positive psychology interventions: A meta-analysis of randomized controlled studies. BMC Public Health. https://doi. org/10.1186/1471-2458-13-119

Breines, J. G., \& Chen, S. (2012). Self-compassion increases self-improvement motivation. Personality and Social Psychology Bulletin, 38(9), 1133-1143. https://doi.org/10.1177/0146167212445599

Buchanan, K., \& Bardi, A. (2010). Acts of kindness and acts of novelty affect life satisfaction. Journal of Social Psychology, 150(3), 235-237. https://doi.org/10.1080/00224540903365554

Cohen, S., Kamarck, To., \& Mermelstein, R. (1983). A global measure of perceived stress. Journal of Health and Social Behavior, 24(4), 385-396.

Cohen, S., \& Williamson, G. (1988). Perceived stress in a probability sample of the United States. In S. Spacapan \& S. Oskamp (Eds.), The social psychology of health (pp. 31-67). Sage.

Crawford, J. R., \& Henry, J. D. (2003). The Depression Anxiety Stress Scales (DASS): Normative data and latent structure in a large non-clinical sample. British Journal of Clinical Psychology, 42(2), 111-131. https://doi.org/10.1348/014466503321903544 
Curry, O. S., Rowland, L. A., Van Lissa, C. J., Zlotowitz, S., McAlaney, J., \& Whitehouse, H. (2018). Happy to help? A systematic review and meta-analysis of the effects of performing acts of kindness on the well-being of the actor. Journal of Experimental Social Psychology, 76(February), 320-329. https://doi.org/10.1016/j.jesp.2018.02.014

Davis, D. E., Choe, E., Meyers, J., Wade, N., Varjas, K., Gifford, A., Quinn, A., Hook, J. N., Van Tongeren, D. R., Griffin, B. J., \& Worthington, E. L. (2016). Thankful for the little things: A metaanalysis of gratitude interventions. Journal of Counseling Psychology, 63(1), 20-31. https://doi. org/10.1037/cou0000107

Diener, E., Emmons, R. A., Larsen, R. J., \& Griffin, S. (1985). The satisfaction with life scale. Journal of Personality Assessment, 49(1), 71-75. https://doi.org/10.1207/s15327752jpa4901_13

Drozd, F., Mork, L., Nielsen, B., Raeder, S., \& Bjørkli, C. A. (2014). Better days-A randomized controlled trial of an internet-based positive psychology intervention. Journal of Positive Psychology, 9(5), 377-388. https://doi.org/10.1080/17439760.2014.910822

Duffy, A., Keown-Stoneman, C., Goodday, S., Horrocks, J., Lowe, M., King, N., Pickett, W., McNevin, S. H., Cunningham, S., Rivera, D., Bisdounis, L., Bowie, C. R., Harkness, K., \& Saunders, K. E. A. (2020). Predictors of mental health and academic outcomes in first-year university students: Identifying prevention and early-intervention targets. Bjpsych Open, 6(3), 1-8. https://doi.org/10.1192/ bjo. 2020.24

Faul, F., Erdfelder, E., Lang, A.-G., \& Buchner, A. (2007). G*Power 3: A flexible statistical power analysis program for the social, behavioral, and biomedical sciences. Behavior Research Methods, 39(2), 175-191.

Feicht, T., Wittmann, M., Jose, G., Mock, A., Von Hirschhausen, E., \& Esch, T. (2013). Evaluation of a seven-week web-based happiness training to improve psychological well-being, reduce stress, and enhance mindfulness and flourishing: A randomized controlled occupational health study. Evidence-Based Complementary and Alternative Medicine. https://doi.org/10.1155/2013/676953

Gandy, W. M., Coberley, C., Pope, J. E., \& Rula, E. Y. (2016). Comparison of the utility of two assessments for explaining and predicting productivity change. Journal of Occupational and Environmental Medicine, 58(1), 69-75. https://doi.org/10.1097/JOM.0000000000000598

Gatt, J. M., Alexander, R., Emond, A., Foster, K., Hadfield, K., Mason-Jones, A., Reid, S., Theron, L., Ungar, M., Wouldes, T. A., \& Wu, Q. (2020). Trauma, resilience, and mental health in migrant and non-migrant youth: An international cross-sectional study across six countries. Frontiers in Psychiatry, 10, 997. https://doi.org/10.3389/fpsyt.2019.00997

Gatt, J. M., Burton, K. L. O., Schofield, P. R., Bryant, R. A., \& Williams, L. M. (2014). The heritability of mental health and wellbeing defined using COMPAS-W, a new composite measure of wellbeing. Psychiatry Research, 219(1), 204-213. https://doi.org/10.1016/j.psychres.2014.04.033

Grant, F., Guille, C., \& Sen, S. (2013). Well-being and the risk of depression under stress. PLoS ONE, 8(7), 1-6. https://doi.org/10.1371/journal.pone.0067395

Hallford, D. J., \& Mellor, D. (2016). Brief reminiscence activities improve state well-being and self-concept in young adults: A randomised controlled experiment. Memory, 24(10), 1311-1320. https:// doi.org/10.1080/09658211.2015.1103875

Hamar, B., Coberley, C., Pope, J. E., \& Rula, E. Y. (2015). Well-being improvement in a midsize employer: Changes in well-being, productivity, health risk, and perceived employer support after implementation of a well-being improvement strategy. Journal of Occupational and Environmental Medicine, 57(4), 367-373. https://doi.org/10.1097/JOM.0000000000000433

Hausmann, L. R. M., Youk, A., Kent Kwoh, C., Ibrahim, S. A., Hannon, M. J., Weiner, D. K., Gallagher, R. M., \& Parks, A. (2017). Testing a positive psychological intervention for osteoarthritis. Pain Medicine (united States), 18(10), 1908-1920. https://doi.org/10.1093/pm/pnx141

Hendriks, T., Schotanus-Dijkstra, M., Hassankhan, A., de Jong, J., \& Bohlmeijer, E. (2020). The efficacy of multi-component positive psychology interventions: A systematic review and meta-analysis of randomized controlled trials. Journal of Happiness Studies, 21(1), 357-390. https://doi.org/10. 1007/s10902-019-00082-1

Higgins, J. P. T., Altman, D. G., Gøtzsche, P. C., Jüni, P., Moher, D., Oxman, A. D., Savović, J., Schulz, K. F., Weeks, L., \& Sterne, J. A. C. (2011). The Cochrane Collaboration's tool for assessing risk of bias in randomised trials. BMJ (online), 343(7829), 1-9. https://doi.org/10.1136/bmj.d5928

Iasiello, M., van Agteren, J., Keyes, C. L. M., \& Cochrane, E. M. (2019). Positive mental health as a predictor of recovery from mental illness. Journal of Affective Disorders, 251, 227-230. https://doi. org/10.1016/j.jad.2019.03.065

Joutsenniemi, K., Kaattari, C., Härkänen, T., Pankakoski, M., Langinvainio, H., Lönnqvist, J., Mattila, A. S., \& Mustonen, P. (2014). E-mail-based exercises in happiness, physical activity and readings: 
A randomized trial on 3274 finns. African Journal of Psychiatry (south Africa). https://doi.org/10. 4172/Psychiatry. 1000140

Krueger, C., \& Tian, L. (2004). A comparison of the general linear mixed model and repeated measures ANOVA using a dataset with multiple missing data points. Biological Research for Nursing, 6(2), 151-157. https://doi.org/10.1177/1099800404267682

Kushlev, K., Heintzelman, S. J., Lutes, L. D., Wirtz, D., Kanippayoor, J. M., Leitner, D., \& Diener, E. (2020). Does happiness improve health? Evidence from a randomized controlled trial. Psychological Science. https://doi.org/10.1177/0956797620919673

Lamers, S. M. A., Westerhof, G. J., Glas, C. A. W., \& Bohlmeijer, E. T. (2015). The bidirectional relation between positive mental health and psychopathology in a longitudinal representative panel study. Journal of Positive Psychology, 10(6), 553-560. https://doi.org/10.1080/17439760.2015. 1015156

Liebenberg, L., \& Moore, J. C. (2018). A social ecological measure of resilience for adults: The RRCARM. Social Indicators Research, 136(1), 1-19. https://doi.org/10.1007/s11205-016-1523-y

Lovibond, P. F., \& Lovibond, S. H. (1995). The structure of negative emotional states: Comparison of the Depression Anxiety Stress Scales (DASS) with the Beck Depression and Anxiety Inventories. Behaviour Research and Therapy, 33(3), 335-343. https://doi.org/10.1016/0005-7967(94)00075-U

Lyubomirsky, S. (2012). Hedonic adaptation to positive and negative experiences. The Oxford Handbook of Stress, Health, and Coping. https://doi.org/10.1093/oxfordhb/9780195375343.013.0011

Martín-María, N., Miret, M., Caballero, F. F., Rico-Uribe, L. A., Steptoe, A., Chatterji, S., \& AyusoMateos, J. L. (2017). The impact of subjective well-being on mortality: A meta-analysis of longitudinal studies in the general population. Psychosomatic Medicine, 79(5), 565-575. https://doi.org/ 10.1097/PSY.0000000000000444

Meléndez Moral, J. C., Fortuna Terrero, F. B., Sales Galán, A., \& Mayordomo Rodríguez, T. (2015). Effect of integrative reminiscence therapy on depression, well-being, integrity, self-esteem, and life satisfaction in older adults. Journal of Positive Psychology, 10(3), 240-247. https://doi.org/10. 1080/17439760.2014.936968

Moskowitz, J. T., Carrico, A. W., Duncan, L. G., Cohn, M. A., Cheung, E. O., Batchelder, A., Martinez, L., Segawa, E., Acree, M., \& Folkman, S. (2017). Randomized controlled trial of a positive affect intervention for people newly diagnosed with HIV. Journal of Consulting and Clinical Psychology, 85(5), 409-423. https://doi.org/10.1037/ccp0000188

Neff, K. D. (2003a). Self-compassion: An alternative conceptualization of a healthy attitude toward oneself. Self and Identity, 2, 85-101. https://doi.org/10.1080/15298860390129863

Neff, K. D. (2003b). The development and validation of a scale to measure self-compassion. Self and Identity, 2(3), 223-250. https://doi.org/10.1080/15298860309027

Parks, A. C., Della Porta, M. D., Pierce, R. S., Zilca, R., \& Lyubomirsky, S. (2012). Pursuing happiness in everyday life: The characteristics and behaviors of online happiness seekers. Emotion, 12(6), 1222-1234. https://doi.org/10.1037/a0028587

Pavot, W., \& Diener, E. (2008). The satisfaction with life scale and the emerging construct of life satisfaction. Journal of Positive Psychology, 3(2), 137-152. https://doi.org/10.1080/174397607017569 46

Pinquart, M., \& Forstmeier, S. (2012). Effects of reminiscence interventions on psychosocial outcomes: A meta-analysis. Aging \& Mental Health, 16(5), 541-558. https://doi.org/10.1080/13607863.2011. 651434

R Core Team. (2020). R: A language and environment for statistical computing. R Foundation for Statistical Computing.

Roberti, J. W., Harrington, L. N., \& Storch, E. A. (2006). Further psychometric support for the 10-item version of the perceived stress scale. Journal of College Counseling, 9(2), 135-147. https://doi.org/ 10.1002/j.2161-1882.2006.tb00100.x

Sanders, C. A., Schueller, S. M., Parks, A. C., \& Howell, R. T. (2019). Understanding long-term trajectories in web-based happiness interventions: Secondary analysis from two web-based randomized trials. Journal of Medical Internet Research. https://doi.org/10.2196/13253

Schotanus-Dijkstra, M., Drossaert, C. H. C., Pieterse, M. E., Boon, B., Walburg, J. A., \& Bohlmeijer, E. T. (2017). An early intervention to promote well-being and flourishing and reduce anxiety and depression: A randomized controlled trial. Internet Interventions, 9(April), 15-24. https://doi.org/ 10.1016/j.invent.2017.04.002

Schueller, S. M., \& Parks, A. C. (2012). Disseminating self-help: Positive psychology exercises in an online trial. Journal of Medical Internet Research, 14(3), 1-11. https://doi.org/10.2196/jmir.1850 
Sin, N. L., \& Lyubomirsky, S. (2009). Enhancing well-being and alleviating depressive symptoms with positive psychology interventions: A practice-friendly meta-analysis. Journal of Clinical Psychology, 65(5), 467-487. https://doi.org/10.1002/jclp.20593

van Buuren, S., \& Groothuis-Oudshoorn, K. (2011). mice: Multivariate imputation by chained equations in R. Journal of Statistical Software, 45(3), 1-67. https://doi.org/10.18637/jss.v045.i03

World Health Organization. (2004). Promoting mental health: Concepts, emerging evidence, practice (summary report).

Zessin, U., Dickhäuser, O., \& Garbade, S. (2015). The relationship between self-compassion and wellbeing: A meta-analysis. Applied Psychology: Health and Well-Being, 7(3), 340-364. https://doi.org/ 10.1111/aphw.12051

Publisher's Note Springer Nature remains neutral with regard to jurisdictional claims in published maps and institutional affiliations. 